\title{
A GENERALIZED ELEMENT OF DECOMPOSITION FOR DOUBLY PERIODIC FUNCTIONS*
}

BY G. D. NICHOLS

1. Introduction. Hermite $\dagger$ has defined a function $f(x)$ to be doubly periodic of the third kind if it is meromorphic and satisfies two periodicity relations of the form

$$
\begin{aligned}
f(x+2 K) & =e^{\alpha x+\beta} f(x), \\
f\left(x+2 i K^{\prime}\right) & =e^{\gamma x+\delta} f(x) .
\end{aligned}
$$

These relations may be transformed into the following ones

$$
\begin{aligned}
f(x+\pi) & =f(x), \\
f(x+\pi \tau) & =e^{2 i(a x+b)} f(x),
\end{aligned}
$$

and it has been shown that the constant $a$ in (1) is an integer and is equal to the excess of the number of poles over the number of zeros of $f(x)$ in a primitive period cell. For our purposes in the following it is helpful to consider elliptic functions and doubly periodic functions of the second kind as special cases of the above, namely, when $a=b=0$, and $a=0$, respectively.

We derive a generalized element of decomposition which by proper specialization of the constants can be used equally well to obtain expansions for doubly periodic functions of the first, second, or third kinds. It should be noted, however, that the method of the present paper fails to yield a decomposition in the case of doubly periodic functions of the third kind having more zeros than poles, that is, when $a<0$, since the convergence of the series representing the integrals for $C_{r}$ in the following is then destroyed. This same difficulty has been met by other writers in discussing the decomposition of doubly periodic functions of the third kind. $\ddagger$ However, Appell has solved this difficulty by interchanging the roles of $x$ and $y$ in his element of decomposition obtained for the case where $a$ is positive.

\footnotetext{
* Presented to the Society, April 11, 1936.

$\dagger$ Hermite, Oeuvres, vol. 3, p. 329 (footnote); Appell, Mémorial des Sciences Mathématiques, fascicule 36 (1929).

¥ See, for example, a paper by Basoco in Acta Mathematica, vol. 57.
} 
The derivation starts from the integral first used by Teixeira* in obtaining a formula for the expansion of doubly periodic functions of the second kind. The method differs from those frequently used in that it is direct and does not assume a priori the existence and form of the desired element of decomposition.

2. Element of Decomposition. Let $f(x)$ be a doubly periodic function satisfying relations (1), where the imaginary part of $\tau$ is positive. To simplify the notation we shall first assume that $f(x)$ has a single pole of order $k$ in each period parallelogram. Let $x=y$ be the affix of this pole in the period parallelogram $A B C D$, where $A B$ coincides with the axis of reals and $C D$ is above $A B$. Then in the neighborhood of this pole,

$$
f(x)=\frac{A_{k}}{(x-y)^{k}}+\cdots+\frac{A_{2}}{(x-y)^{2}}+\frac{A_{1}}{(x-y)}+P(x-y) .
$$

Consider now Teixeira's function,

$$
\Phi(t)=\frac{e^{2 i t}}{e^{2 i t}-e^{2 i x}} f(t) .
$$

Taking the integral of $\Phi(t)$ around $A B C D$ and applying Cauchy's theorem, we obtain the formula

$$
f(x)=\sum_{r=-\infty}^{\infty} C_{r} e^{2 r i x}-i A_{1}+\sum_{s=1}^{k} D_{y}^{(s-1)} \operatorname{ctn}(x-y) \frac{A_{s}}{(s-1) !},
$$

where

$$
\begin{aligned}
C_{r}=\frac{1}{\pi} \int_{A B} f(t) e^{-2 r i t} d t, & (r=0,1,2, \cdots) \\
C_{-r}=\frac{1}{\pi} \int_{D C} f(t) e^{2 r i t} d t, & (r=1,2,3, \cdots)
\end{aligned}
$$

To calculate $C_{r}, r>0$, integrate $f(t) e^{-2 r i t}$ around a sequence of $n$ period parallelograms of width $\pi$, having $A B$ for upper

* Teixeira, Journal für Mathematik, vol. 125 (1901), pp. 301-318; G. D. Nichols, Tôhoku Mathematical Journal, vol. 37 (1934), pp. 252-258; M. A. Basoco, this Bulletin vol. 37 (i931), pp. 111-124, and vol. 38 (1932), pp. 560568. 
boundary and extending in the downward direction. Applying Cauchy's theorem and allowing $n$ to become infinite gives

(4)

$$
\begin{aligned}
& C_{r}= \\
& -2 i \sum_{s=1}^{k} \sum_{n=1}^{\infty} e^{-2 i(a y n+y r+n b)} q^{a n(n+1)+2 r n}(-2 i r-2 i a n)^{s-1} \frac{A_{s}}{(s-1) !},
\end{aligned}
$$

where $q=e^{\pi i r}$. The coefficient $C_{-r}$ is obtained by integrating $f(t) e^{2 r i t}$ around a similar sequence of parallelograms having $C D$ for lower boundary and extending upward. Its value is obtained from that for $C_{r}$ by replacing $i$ with $-i$. For elliptic functions $C_{0}$ cannot be calculated by this method, but for the other two kinds of functions its value is given by (4). The regions of convergence for the $C$ 's will be discussed below in connection with the final result.

It is interesting to note at this point that if we had integrated $f(t) e^{-2 r i t}$ around the period parallelogram $A B C D$, we would have been led to the difference equation

$$
C_{r}-q^{-2 r} e^{2 i b} C_{r-a}=2 i e^{-2 i y r} \sum_{s=1}^{k}(-2 i r)^{s-1} \frac{A_{s}}{(s-1) !},
$$

of which the above value of $C_{r}$ is a solution. The value of $C_{-r}$ could have been obtained in a similar manner.

Substituting the above values for the $C$ 's into (2) gives

$$
\begin{aligned}
f(x)=C_{0}+\sum_{n=-\infty}^{\infty} \sum_{s=1}^{k} q^{a n(n-1)} e^{2 n i b} D_{y}^{(s-1)} e^{2 i y a n} \\
\cdot[\operatorname{ctn}(x-y-n \pi \tau)-\alpha i] \frac{A_{s}}{(s-1) !},
\end{aligned}
$$

where $\alpha=1$ for $n$ zero or positive, $\alpha=-1$ for $n$ negative, and $-I(\pi \tau)<I(x-y)<I(\pi \tau)$.

We may now write our final result as follows:

$$
f(x)=B+\sum \frac{\phi_{k}(x, y, b)}{(k-1) !}
$$

where

$$
\begin{aligned}
\phi_{k}(x, y, b)=A_{1} \psi(x, y, b) & +A_{2} D_{y} \psi(x, y, b)+\cdots \\
& +A_{k} D_{y}^{(k-1)} \psi(x, y, b),
\end{aligned}
$$


and where the summation is extended over all poles of $f(x)$ in a given period parallelogram and where

(6) $\psi(x, y, b)=\sum_{n=-\infty}^{\infty} q^{a n(n-1)} e^{2 n i b} e^{2 i y a n}[\operatorname{ctn}(x-y-n \pi \tau)-\delta i]$

is the desired generalized element of decomposition.

For elliptic functions we take $a=b=0, \delta=0$ for $n$ zero, $\delta=1$ for $n$ positive, and $\delta=-1$ for $n$ negative. $B$ is the usual constant occurring in classical formulas of decomposition and our psifunction (6) is seen to be identical with the zeta-function of Jacobi and Hermite.*

For doubly periodic functions of the second kind $a=B=0$, and $\delta=1$ for all values of $n$. In this case there is the further restriction that $0>I(b)>-I(\pi \tau)$. The element of decomposition obtained from (6) for this class of functions does not seem to have been given hitherto, although it can be checked by means of certain of the results given by Teixeira. $\dagger$ By a slight change in procedure a similar element can be derived which is valid for $I(b)$ positive. For doubly periodic functions of the third kind $b=B=0$ and $\delta=1$ for all values of $n$. Our function (6) for this case is closely related to the chi-function of Appell, $\ddagger$ and can be shown to give the same expansion for $f(x)$. To do this consider $g(x)=\sum_{n=-\infty}^{\infty} q^{a n(n-1)} e^{2 i x a n}$, which satisfies the two relations $g(x+\pi)=g(x), g(x+\pi \tau)=e^{-2 i a x} g(x)$. Hence $f(x) \cdot g(x)$ is an elliptic function and furthermore has the same poles as $f(x)$. The residue at the pole $x=y$ is

$$
\sum_{s=1}^{k} D_{y}^{(s-1)} g(y) \frac{A_{s}}{(s-1) !},
$$

and the sum of all such residues at poles within each period cell is zero. But this sum is exactly the part added to the expansion (5) by the difference between the element (6) and that of Appell.

UnIVERsity of ARKansas p. 115 .

* Appell and Lacour, Principes des Fonctions Elliptiques, 2d edition, 1922,

$\dagger$ Teixeira, loc. cit.

$\ddagger$ Appell and Lacour, Principes des Fonctions Elliptiques, 2d edition, 1922, p. 396; Halphen, Fonctions Elliptiques, vol. 1, p. 468; M. A. Basoco, Acta Mathematica, vol. 57, pp. 95-100. 